Draft Version OCtober 13, 2018

Preprint typeset using $\mathrm{LATE}_{\mathrm{E} X}$ style emulateapj v. 11/10/09

\title{
A VARIABLE BLACK HOLE X-RAY SOURCE IN A NGC 1399 GLOBULAR CLUSTER ${ }^{1}$
}

\author{
I Chun Shin ${ }^{2,3}$, Arunav Kundu ${ }^{2}$, Thomas J. Maccarone ${ }^{4}$, Stephen E. ZepF ${ }^{2}$, \& Tana D. Joseph ${ }^{4}$ \\ Draft version October 13, 2018
}

\begin{abstract}
We have discovered an accreting black hole $(\mathrm{BH})$ in a spectroscopically confirmed globular cluster (GC) in NGC 1399 through monitoring of its X-ray activity. The source, with a peak luminosity of $\mathrm{L}_{X} \simeq 2 \times 10^{39} \mathrm{ergs} \mathrm{s}^{-1}$, reveals an order of magnitude change in the count rate within $\simeq 10 \mathrm{ks}$ in a Chandra observation. The $\mathrm{BH}$ resides in a metal-rich $[\mathrm{Fe} / \mathrm{H}] \simeq 0.2$ globular cluster. After $\mathrm{RZ} 2109$ in NGC 4472 this is only the second black-hole X-ray source in a GC confirmed via rapid X-ray variability. Unlike RZ 2109, the X-ray spectrum of this BH source did not change during the period of rapid variability. In addition to the short-term variability the source also exhibits long-term variability. After being bright for at least a decade since 1993 within a span of 2 years it became progressively fainter, and eventually undetectable, or marginally detectable, in deep Chandra and XMM observations. The source also became harder as it faded. The characteristics of the long term variability in itself provide sufficient evidence to identify the source as a BH. The long term decline in the luminosity of this object was likely not recognized in previous studies because the rapid variability within the bright epoch suppressed the average luminosity in that integration. The hardening of the spectrum accompanying the fading would also make this black hole source indistinguishable from an accreting neutron star in some epochs. Therefore some low mass X-ray binaries identified as NS accretors in snapshot studies of nearby galaxies may also be BHs. Thus the discovery of the second confirmed BH in an extragalactic GC through rapid variability at the very least suggests that accreting BHs in GCs are not exceedingly rare occurences.
\end{abstract}

Subject headings: Galaxies:individual (NGC 1399) — galaxies:star clusters — X-rays:binaries

\section{INTRODUCTION}

The high spatial resolution of X-ray telescopes such as Chandra and XMM-Newton has led to the discovery of large populations of resolved X-ray sources around nearby galaxies. The vast majority of the point sources observed in elliptical galaxies are believed to be lowmass X-ray binaries (LMXBs). Recent studies have established that roughly $40 \%$ of these LMXBs are associated with globular clusters (e.g. Kundu et al. 2007; Sivakoff et al. 2007). This overabundance of LMXBs in globular clusters (GCs), that typically account for less than $\sim 0.1 \%$ of the stellar mass of a galaxy, reflects the importance of stellar dynamical interactions in the dense inner regions of clusters that leads to the enhanced formation of close binaries.

This raises the interesting question of whether some of these LMXBs may be black hole (BH) accretors. There is much interest in black holes residing in globular clusters in part because it is often suggested that GCs are among the most likely hosts of intermediate mass black holes.

\footnotetext{
${ }^{1}$ Based on observations made with the Chandra X-ray Observatory, which is operated by the Smithsonian Astrophysical Observatory for and on behalf of the National Aeronautics Space Administration under contract NAS8-03060 and the NASA/ESA Hubble Space Telescope which is operated by the Association of Universities for Research in Astronomy, Inc., under NASA contract NAS 5-26555.

2 Physics \& Astronomy Department, Michigan State University, East Lansing, MI 48824; e-mail: Stephen.Shih@obspm.fr, akundu@pa.msu.edu, zepf@pa.msu.edu

${ }^{3}$ Current address: Institute of Astronomy, National Tsing Hua University, Hsinchu, Taiwan

${ }^{4}$ School of Physics \& Astronomy, University of Southampton, Southampton, Hampshire SO17 1BJ; e-mail: tjm@astro.soton.ac.uk, tdj1f08@soton.ac.uk
}

On the other hand there have also been suggestions that most black holes are ejected from GCs due to dynamical processes (Sigurdsson \& Hernquist 1993; Kulkarni et al. 1993).

One way to detect a black hole in a GC is to search for a characteristic X-ray signature if the $\mathrm{BH}$ happens to be accreting from a nearby companion. So far no such accreting BHs have been observed in any of the globular clusters in the Milky Way. But Chandra and XMM observations have identified a number of GCs in nearby galaxies that host LMXBs that are brighter than the Eddington luminosity for an accreting neutron star (Angelini et al. 2001; Kundu et al. 2002; Kim et al. 2006). However these bright sources may also represent the superposition of many neutron star LMXBs (e.g. Angelini et al. 2001; Kundu et al. 2007) in globular clusters. Detection of rapid X-ray variability in such sources may be the only definitive way to confirm a black hole accretor with present instruments (e.g. Kalogera et al. 2004).

We have previously discovered the first, and so far only, black hole accretor in a GC through X-ray variability in RZ 2109, a globular cluster in the bright Virgo cluster galaxy NGC 4472 (Maccarone et al. 2007; Shih et al. 2008; Zepf et al. 2008). Identification of a single such candidate leaves open many intriguing questions about the ubiquity of such systems. It is possible that the discovery of a BH in the globular cluster RZ 2109 owes to an extraordinarily fortuitous set of circumstances. In this paper we demonstrate the presence of a black hole in a second extragalactic globular cluster in NGC 1399 through variability in the X-ray flux of a luminous accretor. 
NGC 1399 is the giant elliptical galaxy at the center of the Fornax Cluster. It has a rich and well-studied globular cluster system (e.g. Grillmair et al. 1999; Dirsch et al. 2003) with a bimodal GC metallicity distribution that is typical in such galaxies (e.g. Kundu \& Whitmore 2001). Many joint optical and X-ray studies of NGC 1399 have showed that it has one of the highest fraction LMXBs associated with GCs of any galaxy studied to date. As in other galaxies metal-rich clusters are more likely to host LMXBs than metal-poor ones (Angelini et al. 2001; Kim et al. 2006; Kundu et al. 2007). We describe below the discovery of a black hole in one of the luminous GCs in this galaxy.

\section{OBSERVATIONS AND RESULTS}

\subsection{X-rays}

NGC 1399 has been observed multiple times by both the Chandra and XMM-Newton observatories in the past decade (Table 1). This provides an unique opportunity to trace X-ray activity in LMXBs, and in particular those associated with GCs for the purpose of this study.

We downloaded the archival Chandra and XMMNewton data of NGC 1399 listed in Table 1 and processed it following standard data reduction guidelines using CIAO v4.0 and SAS v8.0, respectively. Intervals of high background were filtered out from the data, and all images were corrected for exposure and vignetting. We adopted the wavelet method of source detection to identify point sources.

We discovered that one of the candidate LMXBs observed during the course of Chandra observation 319 displayed rapid X-ray variability on the timescale of $\sim 10$ ksec (Fig 1). A literature search reveals that this source has been observed by ROSAT-HRI multiple times between 1993 and 1997 and is also listed in the 2XMM catalogue as 2XMM J033831.7-353058:042943 (Paolillo et al. 2002; Liu \& Bregman 2005; Watson et al. 2009). This object was also identified in Kundu et al. (2007) as X-ray source number 157, CXOKMZJ033831.7-353058 (hereafter, the source). The $5.4 \times 10^{38} \mathrm{ergs} \mathrm{s}^{-1} \mathrm{X}$-ray luminosity derived using the average count rate of the source through the entire Chandra observation is brighter than the Eddington luminosity of an accreting neutron star (also see $\S 2.4 .1$ below), suggesting that this may be a black hole candidate. However the source lies outside the HST image of the inner region analyzed in Kundu et al. (2007). Thus, we turn to other data sets to check whether this object is associated with a globular cluster.

\subsection{Optical Counterpart}

We downloaded F606W band HST-ACS images of NGC 1399 from the archive (HST GO proposal 10129) and created an aligned mosaic of the nine independent pointings of the region around the galaxy. We used the Multidrizzle routine based on the drizzle code of Fruchter \& Hook (2002) for this purpose, taking into account the small errors in the default header values. We aligned the HST mosaic to the USNO-A2.0 astrometric system (Monet et al. 1998). Next we identified the point sources corresponding to the spectroscopically confirmed GCs studied by Dirsch et al. (2004). The location of the GCs in the HST mosaic image was used to derive more secure positions for these objects. Finally we spatially aligned the X-ray and optical images in the manner described in Maccarone et al. (2003). The rms of the optical and X-ray source matches was $0.2^{\prime \prime}$.

The variable X-ray source CXOKMZJ033831.7-353058 is coincident with the globular cluster identified as 86:53.0 by Dirsch et al. (2004). The matching radius is only $0.14^{\prime \prime}$ confirming that this object is located in a GC. It is located almost exactly 4' due South of NGC 1399 at $\mathrm{RA}=03: 38: 31.7$ Dec=-35:30:59.21 (J2000). These coordinates are based on the location of the source in the HST image aligned to the USNO system. The host globular cluster has a magnitude and color $\mathrm{R}=22.02 \pm 0.03$ and $\mathrm{C}-\mathrm{R}=2.04 \pm 0.09$ (Dirsch et al. 2004). In the next few sections we examine all the Chandra and XMM-Newton observations of NGC 1399 which covered the position of the source (Table 1).

\subsection{Short Term Variability and Spectral Analysis}

We created background-subtracted lightcurves from the observations in which the source can be detected. As the source is fairly isolated with no obvious X-ray emitters nearby we used an annular region surrounding the object with $\sim 1: 1$ area ratio to determine the backgroundsubtracted count rate. Only the first data set, Chandra observation 319, revealed a rapid short-term variability (see Fig. 1). The count rate declined by an order of magnitude during the first $10 \mathrm{ksecs}$. The source remained faint for the rest of the observation and showed no further evidence of measurable variability. To verify that the decline was not due to an instrumental effect we compared the lightcurves of other X-ray sources in the same CCD (ACIS-S3), and found no similar patterns. In the rest of this paper we consider the first 9,873 s of Chandra Obs ID 319 in which the count rate of the is either high or declining the "bright" phase. The other 46,070 s during which the count rate is at a steady low level is referred to as the "faint" phase.

Spectroscopic analysis of the Chandra data was carried out using the Interactive Spectral Interpretation System (ISIS), version 1.4.9-55. Data were binned within ISIS into groups with signal-to-noise of at least 2.0, and all channels with at least this signal-to-noise within the range from $0.3-8.0 \mathrm{keV}$ were included in the fits. Due to the limited count rate we only attempted to fit two standard models which are commonly used to describe the continuum spectra of X-ray binaries, disk blackbody (DISKBB) and power-law (PO). The foreground absorption was fixed to the Galactic neutral Hydrogen column density $(\mathrm{nH})$ in the direction of NGC $1399, \sim 1.31 \times 10^{20}$ $\mathrm{cm}^{-2}$ (Dickey \& Lockman 1990).

The data were fit using the Gehrels statistic to define the errors (with the errors set to $1+\sqrt{(N+.75)}$, where $N$ is the number of counts in the bin) on the individual bins, as this binning has been shown to provide more reliable fitting to bins with low count numbers. It was found that binning to the more standard signal-to-noise of 5.0 per bin led to too few channels for fitting and binned out real information in the data. The uncertainty estimates for all fits reflect $90 \%$ confidence limits.

The results of the spectral fitting of the bright and faint epochs of the Chandra 319 observations are summarized in Table 2 and plotted in Fig 2. Both the disk blackbody and power law fits reveal a soft spectrum 
for both time periods, with the PO model providing a marginally better fit. This is consistent with a luminous X-ray binary in a very-high state, where the power law component dominates the luminosity (Nowak 1995; McClintock \& Remillard 2006). We note here that some of the inordinately large uncertainty estimates in the normalization of the disk blackbody models, for these and other fits listed in Table 2, are correlated to the large errors in the temperature, but lead to consistent total luminosity.

There is no statistically significant difference between the spectral properties of the source in the two epochs. Using the power-law model the X-ray flux of the source in the energy range of $0.3-8.0 \mathrm{keV}$ is estimated to be $2.8 \times 10^{-14}$ ergs cm ${ }^{-2} \mathrm{~s}^{-1}$ in the bright phase and $2.8 \times 10^{-15}$ in the faint phase. Adopting a distance of $20 \mathrm{Mpc}$ (Tonry et al. 2001) this implies an isotropic luminosity of $1.3 \times 10^{39} \mathrm{ergs} \mathrm{s}^{-1}$ during the bright epoch and $1.3 \times 10^{38} \mathrm{ergs} \mathrm{s}^{-1}$ during the faint one. We note here that the average luminosity quoted in Kundu et al. (2007), and mentioned in $\$ 2.1$ above was calculated using fixed a spectral index $\Gamma=1.7$ for all sources in NGC 1399 due to the difficulty of reliably measuring this parameter for many of the sources superimposed on the bright hot gas in the inner regions.

\subsection{Long Term Variability}

We searched for the source in all of the Chandra and XMM data sets listed in Table 1 . The source was detected in all observations through the middle of 2003 but appears to have subsequently turned off indicating long term variability. The individual observations are briefly discussed below.

\subsubsection{XMM Observation 0055140101}

These XMM observations were taken one year after the Chandra 319 observations discussed above. The source is located on the edge of the XMM-Newton EPIC MOS2 image and is the basis for its detection and listing as 2XMM J033831.7-353058:042943 in the second XMM-Newton Serendipitous Source Catalogue (2XMM, Watson et al. 2009). Following standard data reduction guidelines using SAS v8.0. As in the Chandra analysis we used an annular region surrounding the object with $\sim 1: 1$ area ratio to account for the background.

We fit the XMM data of the source in XSPEC 11.0 using the Gehrels statistic, without binning channels. Within the uncertainties the spectral properties of the source are indistinguishable from the Chandra 319 observations (Table 2). The flux, $F_{X} \simeq 4.2 \times 10^{-14} \mathrm{ergs} \mathrm{cm}^{-2}$ $\mathrm{s}^{-1}$ for the power law fit, is similar to the level of the bright phase of the Chandra 319 observations. Thus the source regained its luminosity within a year of the short term variability observed in the earlier Chandra data. This translates to an isotropic luminosity of $\mathrm{L}_{X} \simeq 2 \times 10^{39}$ $\operatorname{ergs~s}{ }^{-1}$, which is slightly brighter than the estimate from the bright phase of Chandra Obs ID 319, but consistent with previous ROSAT estimates (Liu \& Bregman 2005). However, we note that the short timescale variation in Chandra Obs ID 319 occurred at the very beginning of the observation leaving open the possibility that the source was in fact brighter immediately prior to this observation. Thus we consider the $\mathrm{L}_{X} \simeq 2 \times 10^{39} \mathrm{ergs}$ $\mathrm{s}^{-1}$ luminosity from this XMM observation to be a fairer estimate of the peak brightness of this source.

\subsubsection{XMM Observation 0012830101}

The source was detected in the $10 \mathrm{ks}$ XMM Newton observations of NGC 1399 on 2001-06-27 (Obs ID 0012830101). Given the short exposure and consequently low counts we do not attempt to fit the spectrum. The 2XMM flux of this source is estimated to be $4.1 \times 10^{-14}$ ergs $\mathrm{cm}^{-2} \mathrm{~s}^{-1}$, which implies an isotropic luminosity of $\mathrm{L}_{X} \simeq 1.9 \times 10^{39} \mathrm{ergs} \mathrm{s}^{-1}$. Thus the source appears to have remained as bright as observed in the previous XMM observation obtained 6 months before.

\subsubsection{Chandra Observation 2942}

The source was in the field of view of the Chandra ACIS observation of NGC 1404, taken roughly three years after the Chandra 319 observation. At this time, the spectrum was harder than at earlier epochs (Table 2 ). The X-ray flux in the $0.3-8.0 \mathrm{keV}$ band from the power law fit is $1.9 \times 10^{-14} \mathrm{erg} \mathrm{cm}^{-2} \mathrm{~s}^{-1}$, a factor of $\sim 2$ lower than the previous epoch. However, the harder spectrum and the $\mathrm{kT} \simeq 0.9 \mathrm{keV}$ estimate suggests that the source may have undergone a state transition to a high/soft state as observed in stellar mass black holes (Nowak 1995; McClintock \& Remillard 2006) and may be better described by a disk blackbody model. The flux estimate of $F_{X} \simeq 1 \times 10^{-14} \mathrm{erg} \mathrm{cm}^{-2} \mathrm{~s}^{-1}$ from a disk blackbody fit implies a luminosity of $4.8 \times 10^{38} \mathrm{ergs} \mathrm{s}^{-1}$, and a factor of 4 decline in the brightness from the previous XMM epoch.

\subsubsection{Chandra Observations $4172 \& 4174$}

Both of these observations were part of the Fornax Cluster survey. The source was in the field of view of the Chandra ACIS I3 (4172) or I0 (4174) chips. Although it was detected by CIAO's source detection tool, the count rate in each case was too low $\left(3.06 \times 10^{-4}\right.$ and $\left.1.39 \times 10^{-4} \mathrm{cts} / \mathrm{s}\right)$ to derive meaningful result by fitting the spectrum. Instead we determined the X-ray flux using the simulation tool PIMMS by assuming that the spectral properties were unchanged since the Chandra observation 2942 . The $\mathrm{X}$-ray flux in the $0.3-8.0 \mathrm{keV}$ range is $\simeq 3.7 \times 10^{-15} \mathrm{erg} \mathrm{cm}^{-2} \mathrm{~s}^{-1}$. Thus the luminosity of the source has dropped by another factor of 2 to $1.8 \times 10^{38}$ $\operatorname{ergs~s}^{-1}$ luminosity since the Chandra 2942 observation.

\subsubsection{XMM Observations $0304940101 \& 0400620101$}

The source is not detected in the XMM-Newton observations 0304940101 and 0400620101 . Neither automated routines nor a careful visual inspection revealed any evidence of an object at or near the location of the source. The measured count rate at the position of the source is indistinguishable from that of the nearby background. Using the XMM FLIX tool we estimate an upper limit of $2.7 \times 10^{-14} \mathrm{ergs} \mathrm{cm}^{-2} \mathrm{~s}^{-1}$ on July 30,2005 and $1 \times 10^{-14}$ ergs $\mathrm{cm}^{-2} \mathrm{~s}^{-1}$ on Aug 23, 2006. Thus the source was fainter than $L_{X} \leq 4.8 \times 10^{38} \mathrm{ergs} \mathrm{s}^{-1}$ at this time.

\subsubsection{Chandra Observations 9798 \& 9799}

We also investigate the two Chandra observations 9798 and 9799 aimed at SN2007on. Since they were taken only three days apart, it makes sense to combine the results 
from the analyses of the two data sets to get one flux value/upper limit. The black hole candidate is $4.9^{\prime}$ off axis in these observations, but is undetected in both. We attempt to estimate an upper limit on the flux by determining how many photons are within $2^{\prime \prime}$ of its location. In observation 9798 we find 4 photons from 0.5 to $8.0 \mathrm{keV}$ within that radius, with an expectation value of $0.96 \pm .03$ photons estimated by looking at an offaxis background region. In observation 9799 we find 7 photons, with an expectation value of $1.24 \pm 0.03$. The source is thus marginally detected when the two observations together are considered - there is only a $2 \times 10^{-5}$ probability that 11 photons will be detected given a background expectation value of 2.2 photons. However, if one considers the trials incurred by checking all positions on the ACIS-S3 chip the detection is not statistically significant.

One can also make an estimate of the upper limit to the luminosity of the source during these two observations, given that the source is marginally detected, with 11 photons seen in 40 ksecs. The $99 \%$ confidence level upper limit on the underlying count rate is then 20.5 photons, of which 18.3 would be expected to be source photons. This translates to a count rate of $4.6 \times 10^{-4}$ photons $\mathrm{sec}^{-1}$. Assuming the spectrum was unchanged since the Chandra 2942 observations this corresponds to a flux limit of $4.6 \times 10^{-15} \mathrm{ergs} \mathrm{cm}^{-2} \mathrm{~s}^{-1}$ and a luminosity upper limit $L_{X} \leq 2.2 \times 10^{38} \mathrm{ergs} \mathrm{s}^{-1}$.

\subsubsection{Chandra Observation 9530}

The source was in the field of view of the Chandra 9530 observation of NGC 1399 obtained on 2008-06-08. No object is detected at the location of the source with the probability of false detection set to $10^{-6}$. However there is a marginal detection when the false source probability is relaxed to $10^{-4}$. This yields 13 background subtracted counts during the course of this $60 \mathrm{ks}$ observation. Although is insufficient to fit a spectrum, we can estimate the luminosity by comparing the count rate to previous Chandra observations. If the source had a soft spectrum similar to that observed on 2001-1-18 then scaling the count rate to the Chandra 319 observations yields a luminosity of $L_{X} \leq 4.1 \times 10^{37} \mathrm{ergs} \mathrm{s}^{-1}$. If on the other hand it retained the harder spectral shape of the 20032-13 Chandra 2942 detection, scaling the count rate to that observation estimates a luminosity of $L_{X} \leq 4.8 \times 10^{37}$ $\operatorname{ergs~s}^{-1}$. Thus, irrespective of the detils of the spectrum the source was $L_{X} \leq 4.8 \times 10^{37} \mathrm{ergs} \mathrm{s}^{-1}$ or fainter at this epoch. This is fainter than the upper limit of the luminosities derived from the previous observations in which the source was not detected, and confirms that it faded since the 2003 Chandra detection. we also note that a luminosity of $L_{X} \leq 4.8 \times 10^{37} \mathrm{ergs} \mathrm{s}^{-1}$ is typical of bright neutron star accretors. There is a high probability of such an object being associated with a high mass, metalrich globular cluster such as the one being studied here (Kundu et al. 2007). Thus the black hole accretor itself may in face be fainter than the luminosity estimated from the Chandra 9530 observation.

\subsection{Discussion and Analysis}

We have confirmed that the X-ray source previously identified as CXOKMZJ033831.7-353058
Kundu et al. 2007) and 2XMM J033831.7353058:042943 (Watson et al. 2009) is associated with the spectroscopically confirmed globular cluster 86:53.0 (Dirsch et al. 2004) in NGC 1399. We have examined the X-ray activity of the source over a period of 8 years using archival Chandra and XMM-Newton data.

Between Jan 2000 and Feb 2003 the X-ray luminosity of the source was above $10^{39} \mathrm{ergs} \mathrm{s}^{-1}$, based on an estimated distance of $20 \mathrm{Mpc}$ to NGC 1399 (Tonry et al. 2001). Earlier ROSAT observations sampling various epochs between 1993 and 1997 estimated a similar luminosity of $2 \times 10^{39} \mathrm{ergs} \mathrm{s}^{-1}$ (Liu \& Bregman 2005). This is well beyond the Eddington luminosity of spherical accretion on to a neutron star and places the source into the category of an ultra luminous X-ray source.

During the course of the first Chandra observation in this analysis (Obs ID 319 in Jan 2000) the source revealed a rapid short-term decline in the count rate within $10 \mathrm{ksecs}$, demonstrating that the source is not a superposition of multiple neutron star binaries and confirming that it is a black hole. After RZ 2109 in NGC 4472 (Maccarone et al. 2007) this is only the second BH X-ray accretor positively identified through short term variations in the count rate. Curiously both sources revealed roughly order of magnitude declines over similar time periods of $\simeq 10 \mathrm{ks}$.

But the parallels appear to end there. The decline in the count rate in RZ 2109 was primarily in the soft X-ray band, indicating a change in the X-ray spectrum consistent with foreground absorption and not a variation in the inherent X-ray luminosity of the source (Shih et al. 2008). On the other hand there is no statistical difference in the spectrum of CXOKMZJ033831.7-353058 between the bright and faint phases in the Chandra 319 observations (Table $2 \&$ Fig. 2). This suggests that either the luminosity of the source changed during the course of the Chandra319 observation or that a portion of the X-ray emitting region was obscured by an eclipsing event.

The NGC 1399 BH also reveals long term variability. Other than a portion of the Chandra Obs ID 319 integration the sources remained brighter than $10^{39}$ ergs $\mathrm{s}^{-1}$ from the 1993 ROSAT observations through the 2001 XMM-Newton observations. Thereafter it became progressively fainter. It was not detected in XMM and Chandra observations between 2005 and 2007. A $L_{X} \leq 4.8 \times 10^{37}$ ergs s$^{-1}$ source was marginally detected in a deeper 2008-06-08 Chandra observation. However such a luminosity is typical of bright neutron star accretors ad there is a high probability of such an object being associated with the host globular cluster of this source. The black hole accretor itself may in fact be even fainter than $L_{X}=4.8 \times 10^{37} \mathrm{ergs} \mathrm{s}^{-1}$ at this point.

The Chandra 2942 observation from 2003 which showed the first clear evidence of a long term decline in the luminosity has a spectral index of $\Gamma=1.3$, considerably harder than the $\Gamma=2.4-2.9$ spectrum at earlier epochs. This suggests that the source underwent a state transition from a very-high state where the power law component dominates to a high/soft state where the emission is primarily thermal (Nowak 1995; McClintock \& Remillard 2006). Such state changes accompanying luminosity variations in a $\mathrm{GC} \mathrm{BH}$ points to 
some interesting issues about identifying and quantifying BHs in globular clusters.

$\mathrm{BH}$ candidates in nearby galaxies (and their GC systems) are identified on the basis of their higher luminosities and softer spectra as compared to NS accretors. However, the hardening of the spectrum of CXOKMZJ033831.7-353058 as it faded places both its luminosity and its spectrum in the range of typical neutron star LMXBs $\left(\mathrm{L}_{X} \leq 10^{38} \mathrm{ergs} \mathrm{s}^{-1}, \Gamma \simeq 1.7\right)$ in the latter epochs of our survey. Thus an unrecognized fraction of the LMXBs in GCs tagged as neutron star systems in snapshot observations of extragalactic systems are likely $\mathrm{BH}$ accretors.

The long term variability of CXOKMZJ033831.7353058 itself provides strong evidence that the source is a black hole. The source was consistently in the ultraluminous regime for at least a decade prior to 2003, before fading relatively rapidly within a span of less than two years. If this object were a superposition of multiple close to Eddington limited neutron star LMXBs it is highly unlikely that they would all conspire to change luminosity within such a short time period. Moreover, given the fact that this is similar to the light travel time across the core of a typical cluster (where neutron star LMXBs are expected to reside) the degree of coincidence required is even more unlikely. The fact that a confirmed $\mathrm{X}$-ray bright black hole system in a GC undergoes physical changes similar to those seen in stellar mass BHs in the MW on the timescales of years is encouraging in terms of identifying and studying the demographics BHs in GCs. It indicates that BHs can be securely identified by monitoring on month-to-year scales without requiring fortuitous occurances of short term variations within a single observation.

There have been previous attempts to detect BHs in elliptical galaxies by such monitoring (e.g. Irwin 2006; Brassington et al. 2008). However, the only likely BHs identified using this technique (Fabbiano et al. 2006; Sivakoff et al. 2008) have been field sources that are not associated with GCs. It is particularly interesting that Irwin (2006) which studied NGC 1399 using a subset of this data set did not recognize the transient nature of CXOKMZJ033831.7-353058. The luminosity of the source varied by about an order of magnitude between the (bright phase of the) Chandra 319 observations and Chandra 4172 observations that bookend the Irwin (2006) analysis. This can likely be attributed averaging across the short term variation in the first epoch which leads to an underestimate of the consistently higher luminosities observed over a decade. We note that the luminosity quoted in our own Kundu et al. (2007) study similarly underestimated the brightness of CXOKMZJ033831.7-353058 in the Chandra 319 integration for the same reason. Given that at least four accreting BH systems in elliptical galaxies (Fabbiano et al. 2006; Maccarone et al. 2007; Brassington et al. 2010, and this source) are known to show short term X-ray flux variability (accompanied by spectral changes in some cases) this strongly argues that careful analysis of both short and long term variations of potential $\mathrm{BH}$ candidates must be carried out in concert if more $\mathrm{BHs}$ are to be identified by this method.

Another feature of accreting BHs in globular clusters may be the presence of optical emission lines. The optical spectrum of RZ 2109 reveals broad, $\simeq 2000 \mathrm{~km} \mathrm{~s}^{-1}$ wide, bright $[\mathrm{O} \mathrm{III}]$ emission associated with the $\mathrm{BH}$ (Zepf et al. 2008). Irwin et al. (2010) argue that another ultraluminous X-ray source in a globular cluster in the inner region of NGC 1399 is a black hole on the basis of $\simeq 75 \mathrm{~km} \mathrm{~s}^{-1}[\mathrm{O}$ III] and [N II] lines. An optical spectrum of the host GC of our source taken with the SOAR optical telescope in 2009 shows no evidence of emission lines. A previous 2-hour Magellan/IMACS optical spectrum of this globular cluster taken in November 2006 (Jimmy Irwin, private communication) also reveals no emission lines. However we note, on the basis of our X-ray monitoring, that the X-ray source had faded considerably before either set of optical spectra were obtained.

The host GC of CXOKMZJ033831.7-353058 also differs from RZ 2109 in NGC 4472 in important ways. While RZ 2109 is a metal-poor cluster, 86:53.0 in NGC 1399 (Dirsch et al. 2004) has a color of $\mathrm{C}-\mathrm{R}=2.04 \pm 0.09$. This places it at the reddest, and hence the most metal-rich end of the metal-rich subpopulation of GCs (Dirsch et al. 2004). Based on the calibration of Lee et al. (2008) this translates to a supersolar metallicity of $[\mathrm{Fe} / \mathrm{H}] \simeq 0.2$ dex. RZ 2109 is also one of the most luminous, and hence most massive GCs in NGC 4472. While 86:53.0 is also among the largest GCs in NGC 1399, unlike RZ 2109 it is not at the tail end of the luminosity function. In quantitative terms RZ 2109 is about 2.6 magnitudes brighter than the peak of the globular cluster luminosity function while 86:53.0 in NGC 1399 is only 1.3 magnitudes brighter than the peak. In other words 86:53.0 is about one-third the $2 \times 10^{6} \mathrm{M}_{\odot}$ mass of RZ 2109 (Zepf et al. 2008).

Our discovery of the second confirmed $\mathrm{BH}$ accretor in a globular cluster on the basis of X-ray variability indicates that the discovery of RZ 2109 in NGC 4472 was not due to a particularly fortuitous configuration in that system and provides encouraging evidence that more such $\mathrm{BHs}$ can be identified by monitoring the X-ray activity. Further studies of temporal variability of luminous X-ray sources in nearby galaxies are needed to establish reliable statistics on the ubiquity of black holes in globular clusters.

ICS, SEZ and AK acknowledge support from NASA through ADP grant NNX08AJ60G and Chandra grant AR8-9011X. AK was also supported by NASA LTSA grant NAG 5-12975. T.J. acknowledges support from a Stobie-SALT studentship, funded jointly by the NRF of South Africa, the British Council and Southampton University. We thank the referee Jimmy Irwin for providing helpful comments that improved this paper.

\section{REFERENCES}

Angelini, L., Loewenstein, M., \& Mushotzky, R. F. 2001, ApJ, 557, L35
Arnaud, K.A., 1996, Astronomical Data Analysis Software and Systems V, eds. Jacoby G. and Barnes J., p17, ASP Conf. Series volume 101 
Brassington, N. J., et al. 2008, ApJS, 179, 142

Brassington, N. J., et al. 2010, arXiv:1003.3236

Colbert, E. J. M., Heckman, T. M., Ptak, A. F., Strickland, D. K., \& Weaver, K. A. 2004, ApJ, 602, 231

Dickey, J. M., \& Lockman, F. J. 1990, ARA\&A, 28, 215

Dirsch, B., Richtler, T., Geisler, D., Forte, J. C., Bassino, L. P., \& Gieren, W. P. 2003, AJ, 125, 1908

Dirsch, B., et al. 2004, AJ, 127, 2114

Fabbiano, G., et al. 2006, ApJ, 650, 879

Fruchter, A. S., \& Hook, R. N. 2002, PASP, 114, 144

Grillmair, C. J., Forbes, D. A., Brodie, J. P., \& Elson, R. A. W. 1999, AJ, 117, 167

Irwin, J. A. 2006, MNRAS, 371, 1903

Irwin, J. A., Brink, T. G., Bregman, J. N., \& Roberts, T. P. 2010, ApJ, 712, L1

Kalogera, V., King, A. R., \& Rasio, F. A. 2004, ApJ, 601, L171

Kim, E., Kim, D.-W., Fabbiano, G., Lee, M. G., Park, H. S., Geisler, D., \& Dirsch, B. 2006, ApJ, 647, 276

King, A. R., \& Ritter, H. 1998, MNRAS, 293, L42

Kulkarni, S. R., Hut, P., \& McMillan, S. 1993, Nature, 364, 421

Kundu, A., Maccarone, T. J., \& Zepf, S. E. 2002, ApJ, 574, L5

Kundu, A., Maccarone, T. J., \& Zepf, S. E. 2007, ApJ, 662, 525

Kundu, A., \& Whitmore, B. C. 2001, AJ, 121, 2950
Lee, M. G., Park, H. S., Kim, E., Hwang, H. S., Kim, S. C., \& Geisler, D. 2008, ApJ, 682, 135

Liu, J.-F., \& Bregman, J. N. 2005, ApJS, 157, 59

Maccarone, T. J., Kundu, A., \& Zepf, S. E. 2003, ApJ, 586, 814

Maccarone, T.J., Kundu, A., Zepf, S.E., \& Rhode, K.L. 2007 Nature, 445, 183

McClintock, J. E., \& Remillard, R. A. 2006, Compact stellar X-ray sources, 157

Monet, D., \& et al. 1998, VizieR Online Data Catalog, 1252, 0

Nowak, M. A. 1995, PASP, 107, 1207

Paolillo, M., Fabbiano, G., Peres, G., \& Kim, D.-W. 2002, ApJ, 565,883

Remillard, R. A., \& McClintock, J. E. 2006, ARA\&A, 44, 49

Shih, I. C., Maccarone, T. J., Kundu, A., \& Zepf, S. E. 2008, MNRAS, 386, 2075

Sigurdsson, S., \& Hernquist, L. 1993, Nature, 364, 423

Sivakoff, G. R., et al. 2007, ApJ, 660, 1246

Sivakoff, G. R., et al. 2008, ApJ, 677, L27

Tonry, J. L., et al. 2001, ApJ, 546, 681

Watson, M. G., et al. 2009, A\&A, 493, 339

Zepf, S. E., et al. 2008, ApJ, 683, L139 
TABLE 1

Chandra and XMM-Newton Observations of the Source

\begin{tabular}{llllll}
\hline Date & Observatory & Obs ID & Instrument & Effective Exposure Time & Detection \\
\hline 2000-01-18 & Chandra & 319 & ACIS-S3 & $\begin{array}{l}55,943 \mathrm{~s} \\
\text { (bright:9,873s; faint:46,070s) }\end{array}$ & yes \\
& & & & $47,080 \mathrm{~s}$ & yes \\
$2001-01-07$ & XMM & $0055140101^{1}$ & EPIC-MOS2 & yes \\
$2001-06-27$ & XMM & 0012830101 & EPIC-MOS2, \& PN & $10,085 \mathrm{~s}$ & yes \\
$2003-02-13$ & Chandra & 2942 & ACIS-S3 & $29,420 \mathrm{~s}$ & yes \\
$2003-05-26$ & Chandra & 4172 & ACIS-I3 & $44,503 \mathrm{~s}$ & yes \\
$2003-05-28$ & Chandra & 4174 & ACIS-I0 & $45,672 \mathrm{~s}$ & no \\
$2005-07-30$ & XMM & 0304940101 & EPIC-MOS2, PN & $20,007 \mathrm{~s}$ & no \\
$2006-08-23$ & XMM & 0400620101 & EPIC all ${ }^{2}$ & $73,040 \mathrm{~s}$ & no \\
$2007-12-24$ & Chandra & 9798 & ACIS-S3 & $18,302 \mathrm{~s}$ & no \\
$2007-12-27$ & Chandra & 9799 & ACIS-S3 & $21,288 \mathrm{~s}$ & yes \\
$2008-06-08$ & Chandra & 9530 & ACIS-S3 & $59,348 \mathrm{~s}$ & \\
& & & & \\
\hline
\end{tabular}

1 2XMM catalogue (Watson et al. 2009).

2 MOS1, MOS2 \& PN cameras.

3 The luminosity of this marginal detection is lower than the upper limits implied in the previous nondetections, and is in the range of typical neutron star accretors. See text for details.

TABLE 2

Spectral Fitting Results and Flux Estimates

\begin{tabular}{|c|c|c|c|c|c|}
\hline \multirow[b]{2}{*}{ Date } & \multirow[b]{2}{*}{ Obs. ID/Epoch } & \multicolumn{2}{|c|}{ Disk Blackbody } & \multirow[b]{2}{*}{$\chi_{\nu}^{2}$} & \multirow[b]{2}{*}{$\begin{array}{l}\text { Unabsorbed Flux } \\
\text { ergs } \mathrm{cm}^{-2} \mathrm{~s}^{-1}\end{array}$} \\
\hline & & $\begin{array}{l}\mathrm{kT}_{\text {in }} \\
(\mathrm{keV})\end{array}$ & Norm. & & \\
\hline $2000-1-18$ & Chandra 319 (bright) & $0.29 \pm_{0.09}^{0.2}$ & $0.17 \pm_{0.2}^{2}$ & 0.44 & $1.9 \times 10^{-14}$ \\
\hline $2000-1-18$ & Chandra 319 (faint) & $0.21 \pm_{0.1}^{0.3^{9}}$ & $0.077 \pm 0$ & 0.62 & $2.1 \times 10^{-15}$ \\
\hline 2001-1-07 & XMM 0055140101 & $0.42 \pm_{02}^{8: 3}$ & $0.061 \pm_{0.06}^{0: 60}$ & 0.26 & $3.3 \times 10^{-14}$ \\
\hline $2003-2-13$ & Chandra 2942 & $0.90 \pm_{0.5}^{3.2}$ & $7.74 \pm_{8}^{19000} \times 10^{-4}$ & 0.66 & $1.0 \times 10^{-14}$ \\
\hline Date & Obs. ID/Epoch & Powe & $\frac{\text { Law }}{\text { Norm. }}$ & $\chi_{\nu}^{2}$ & $\begin{array}{l}\text { Unabsorbed Flux } \\
\text { ergs } \mathrm{cm}^{-2} \mathrm{~s}^{-1}\end{array}$ \\
\hline $2000-1-18$ & Chandra 319 (bright) & $2.42 \pm_{0.6}^{0.7}$ & $5.81 \pm_{2}^{2} \times 10^{-6}$ & 0.43 & $2.8 \times 10^{-14}$ \\
\hline $2000-1-18$ & Chandra 319 (faint) & $2.93 \pm_{1}^{2.0}$ & $5.53 \pm_{4}^{4} \times 10^{-7}$ & 0.31 & $2.8 \times 10^{-15}$ \\
\hline 2001-1-07 & XMM 0055140101 & $2.47 \pm_{0.6}^{0.9}$ & $8.91 \pm_{4}^{4} \times 10^{-6}$ & 0.29 & $4.2 \times 10^{-14}$ \\
\hline $2003-2-13$ & Chandra 2942 & $1.33 \pm_{1}^{1} \cdot 6$ & $2.21 \pm_{1}^{0.9} \times 10^{-6}$ & 0.60 & $1.9 \times 10^{-14}$ \\
\hline
\end{tabular}




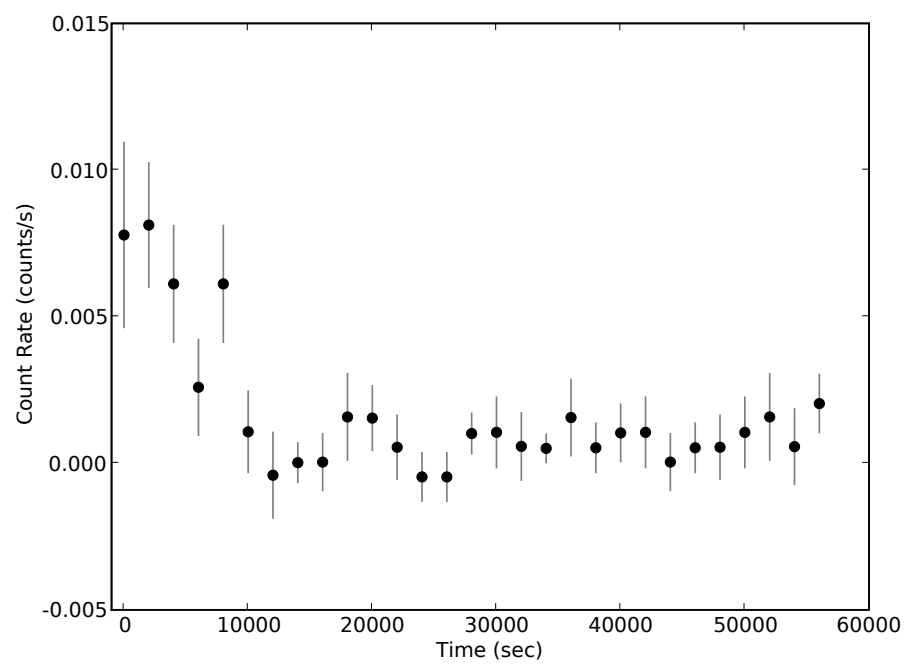

FIG. 1. - The Chandra ACIS-S3 binned lightcurve of the globular cluster X-ray source in NGC 1399 from the 2000-1-18 Obs ID 319 obervations. Photons in the energy 0.3 to $8.0 \mathrm{keV}$ are incuded, and the count rate is averaged over 2,000s.
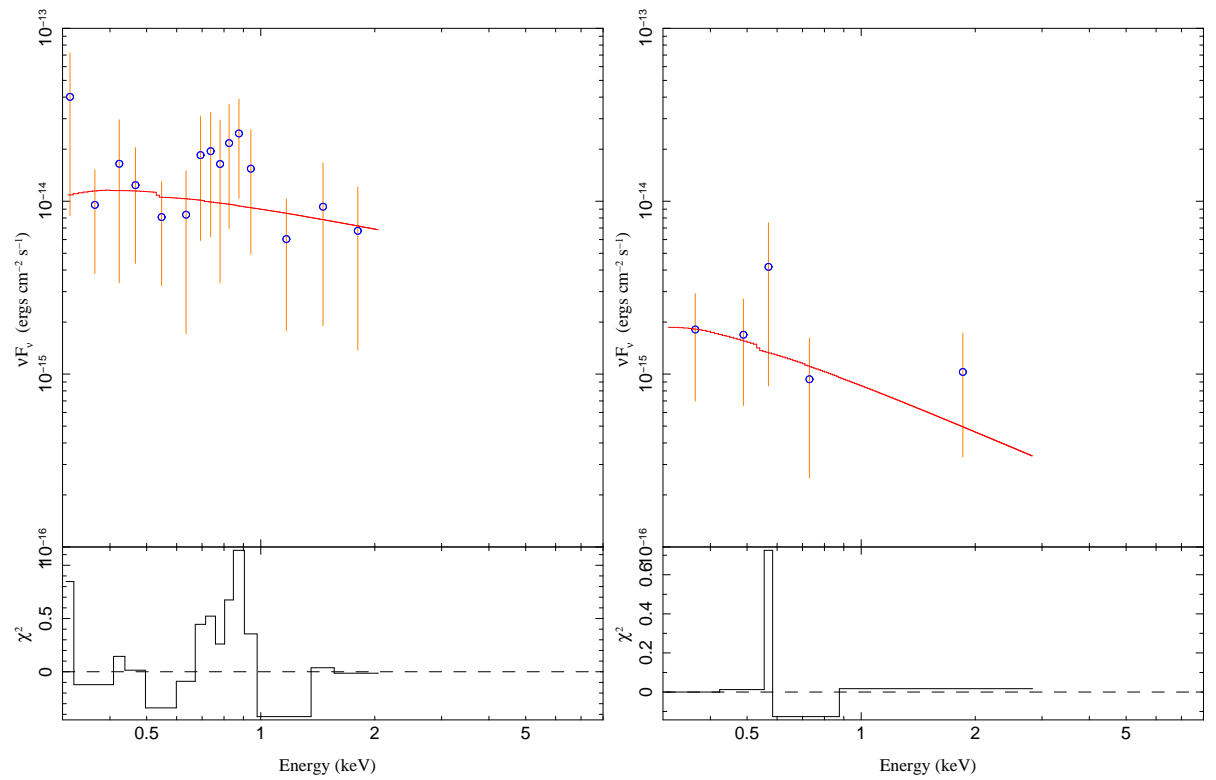

FIG. 2.- The energy density distribution of CXOKMZJ033831.7-353058 during the bright (left) and faint (right) phase of the Chandra Obs ID 319 observations. The best fit powerlaw models and residuals are also shown. There is no obvious difference in the spectrum. 